\title{
Advances in the Inspection of Unpiggable Pipelines
}

\author{
George H. Mills *, Andrew E. Jackson and Robert C. Richardson \\ School of Mechanical Engineering, University of Leeds, Leeds LS2 9JT, UK; A.E.Jackson@Leeds.ac.uk (A.E.J.); \\ R.C.Richardson@Leeds.ac.uk (R.C.R) \\ * Correspondence: G.H.Mills@leeds.ac.uk
}

Received: 2 October 2017; Accepted: 23 November 2017; Published: 29 November 2017

\begin{abstract}
The field of in-pipe robotics covers a vast and varied number of approaches to the inspection of pipelines with robots specialising in pipes ranging anywhere from $10 \mathrm{~mm}$ to $1200 \mathrm{~mm}$ in diameter. Many of these developed systems focus on overcoming in-pipe obstacles such as T-sections and elbows, as a result important aspects of exploration are treated as sub-systems, namely shape adaptability. One of the most prevalent methods of hybridised locomotion today is wall-pressing; generating traction using the encompassing pipe walls. A review of wall-pressing systems has been performed, covering the different approaches taken since their introduction. The advantages and disadvantages of these systems is discussed as well as their effectiveness in the inspection of networks with highly varying pipe diameters. When compared to unconventional in-pipe robotic techniques, traditional full-bore wall-pressing robots were found to be at a disadvantage.
\end{abstract}

Keywords: in-pipe; review; locomotion

\section{Introduction}

Pipeline networks transport fluids such as oil, gas, water, and sewage between key locations through an estimated total of 2.5 million $\mathrm{km}$ (2.2 million miles) of global infrastructure [1]. Failure to adequately inspect and replace pipes results in pipe failure and subsequent loss of fluid transport, environmental damage, large excavations resulting in transport delays and air pollution. Most of the worlds pipelines are relatively easily inspected using advanced Pipeline Inspection Gauges or 'PIGs'; passive devices placed into the pipe and driven by the flow of the transported fluid. However, PIGs are uncontrollable and unable to adapt to sharp changes in pipe direction and diameter, making complex pipe infrastructure impossible to inspect. It is estimated that just $0.5 \%$ of pipe networks are inaccessible to conventional 'PIGGING' technology, the remaining $99.5 \%$ generally consisting of large bore, straight piggable lines. Whilst this proportion may seem low, the remaining 12,500 km represents the most valuable pipes in the network; Above Ground Installations (AGI's). Many of these unpiggable networks are now reaching the end of their design lives and are due for replacement, however with the condition of their interior walls unknown it is impossible to tell which pipes should take replacement priority. It has been estimated that through the use of advanced inspection techniques, savings made from unnecessary pipeline replacement could be equivalent to $£ 14,000$ per $\mathrm{km}$ a year [2].

\subsection{Pipe Bends and Joints}

Unpiggable pipe networks vary in diameter range, material, and fluid type and can be joined in various methods and configurations. Categorised pipe joint configurations are shown in Figure 1. Horizontal sections (Figure 1A) are considered the baseline for in-pipe complexity, any in-pipe robot should be able to navigate these. Configurations B to F are more complex, passing through them requires advanced motion planning techniques. 


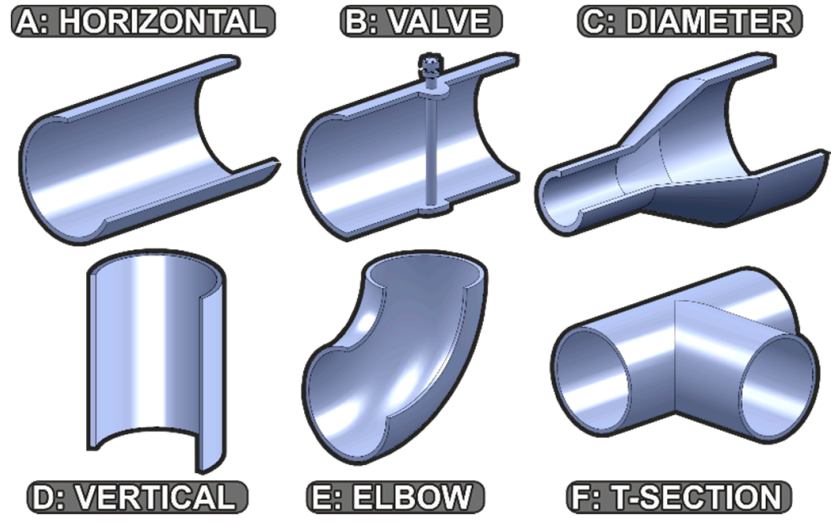

Figure 1. The most commonly encountered in-pipe bends and joints in networks.

Valves, are particularly difficult as designs such as plug valves (Figure 1B) can split the cross-section in two hindering full-bore robots. Changes in diameter (Figure 1C) are a common occurrence in unpiggable systems, many robots take measures to prepare for this obstacle specifically. Vertical sections (Figure 1D) require a traction method that must also overcome gravity. Elbows (Figure 1E) are very commonly encountered and are often described in terms of their bend radius; lower radius bends are tighter harder to navigate. T-Sections (Figure 1F) are extremely challenging obstacles due to their lack of wall support; only sophisticated robotic platforms can navigate these [3]. Each of these in-pipe obstacles can be found in any orientation and possibly even back-to-back e.g., encountering two consecutive bends. Developing a single robot to solve all of these problems in a wide range of diameters is currently unheard of and often requires a fleet of multiple systems in different class sizes [4]. In this review significant robots that have furthered the research field will be presented. Current state-of-the-art methods of in-pipe travel and inspection are discussed as well as the future abilities of in-pipe robots. By analysing the barriers facing current technology and the methods being employed to overcome them, breakthroughs can be made towards universal in-pipe inspection. This review addresses in particular the problems surrounding shape adaptability, fleets, and system classes and their role in universal pipe inspection.

\subsection{Robotic in-Pipe Locomotion}

In-pipe inspection robots have the potential to inspect the condition of these vital assets by manoeuvring through the network. This is no simple feat, and one of the first challenges faced is the generation of traction within the environment. Many potential robotic solutions have been proposed to inspect these 'unpiggable' pipelines, all of which utilised one of the in-pipe traction methods, presented in Figure 2. These traction methods are; Gravity (Figure 2A), reliance on gravity alone restricts vehicles to only horizontal and lightly inclined pipes. Wall-Pressing (Figure 2B), using the reaction force from the enclosed walls, usually in combination with diametric adaption mechanisms. Wall adhesion (Figure 2C), utilising ferrous pipelines to produce a reaction force. Fluid Flow (Figure 2D), utilising the transport medium to move usually in combination with a passive PIG or propeller device. Through the combination of these traction methods and the locomotion elements presented in Figure 2 specialised hybrid in-pipe systems can be created.

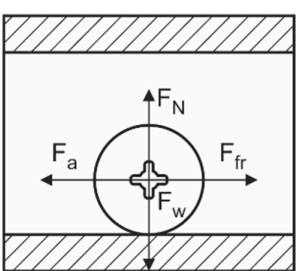

A. Gravity

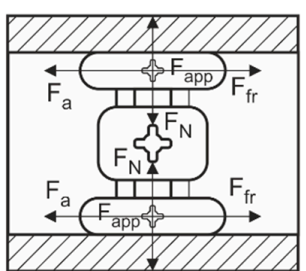

B. Wall-Press

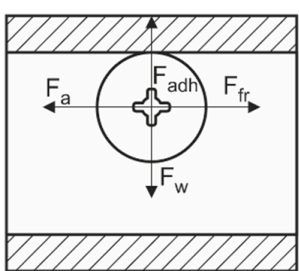

C. Adhesion

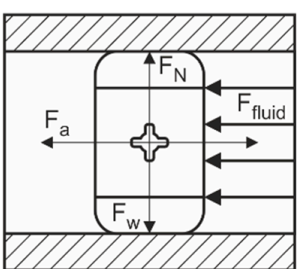

D. Fluid-Flow

Figure 2. In-pipe traction method free body diagrams. 
Initially when issues surrounding in-line inspection of unpiggable pipelines became apparent, traditional gravity based exploration robots were re-purposed for the task [5]. These robots were generally track or wheel based and built to handle rough terrain, they were not well suited to the pipeline environment and could explore only horizontal sections and gentle inclines. Although these systems performed adequately in large bore sewage and water based networks. It wasn't until robotic systems developed advanced methods of in-pipe traction such as wall-pressing, adhesion through magnetics, and fluid flow that the more complex pipeline configurations could be explored. Using the methods of traction in Figure 2 as a design basis, many variations of in-pipe locomotion have been created to solve in-pipe tasks. Figure 3 shows the different systems classified by their locomotion mechanism into the eight types labelled. PIGs (Figure 3A) are transport fluid driven devices, although very effective in horizontal pipes they cannot be controlled in complex networks. Wheeled robots (Figure 3B) are the simplest method of in-pipe locomotion and can be used in combination with many other element types. Tracked robots (Figure 3C), also known as caterpillars, are used as an alternative to wheeled systems, their large surface contact area generates high friction and reduce chances of losing wall contact. Screw robots (Figure 3D) use a spiral inspection path, they perform well in vertical sections and are resistant to slip due to their angled approach, even against an in-pipe flow. Snake robots (Figure 3E) take advantage of the length of the pipe, they are generally modular and adaptable to many in-pipe environments. Inchworm robots (Figure 3F) are slower than other types but can generally carry higher payloads due to their need for high wall-traction forces, useful in industrial transport tasks where speed is unimportant. Propeller based robots (Figure 3G) use transported fluid medium to navigate pipelines and have the advantage of not relying on walls for any movement, however they cannot move in offline systems without fluid. Walking robots (Figure 3H) use legs with multiple degrees of freedom (D.O.F) to move, their end effectors have low surface areas, useful in cutting through in-pipe wall contaminants.


Figure 3. The eight main elements of in-pipe robotic locomotion (A-H).

These basic locomotion elements are more often than not used in conjunction with another, forming a hybrid system. An example of a hybrid system would be an articulated snake robot that uses caterpillar tracks to move, or a screw robot that using wheels for rotation. Of the 234 systems reviewed only $18 \%$ were robots that used just one type, with the rest combining two or more. Wall-pressing is the most popular method of generating in-pipe traction, with $44 \%$ of robotic research systems applying it. These systems mostly consist of a chassis that is kept concentric with the pipe using some form of locomotion method or 'plane' of contact with the wall. These planes are normally tracks or wheel subsystems that are mounted perpendicular to the chassis. Wall-press designs using varying numbers of planes, each with set-up having their own advantages and disadvantages as shown in Figure 4 . 
A great advantage of having a concentric chassis is the distance to each contact plane is constant and methods of pipe diameter adaptability can be centrally located. The parallelogram mechanism and pantograph scissor mechanisms are among the most commonly used in wall-pressing in-pipe robotics. These mechanisms perform well because the planes of contact remain parallel with the chassis of the robot and if centred properly, the inner wall of the pipe. Although they have many variations the most common use the rotation of a central lead screw in combination with a bar linkage design to achieve both traction, and size adaptability.
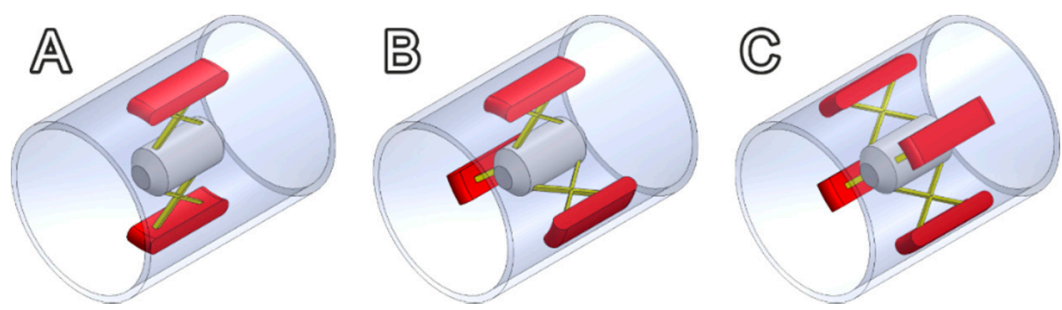

Figure 4. Examples of wall-pressing robots with; (A): 2, (B): 3, and (C): 4 plane designs.

\section{Review of in-Pipe Locomotion Systems}

An in-depth analysis of in-pipe robot publications over the last 30 years has been presented in Figure 5. Categorised by locomotion type and year published it gives a high level overview of the trends in the development of in-pipe inspection robots based on 234 published systems. The literature search was performed using the keywords; in-pipe, pipe, tube, system, robot, platform, in combination with locomotion and traction methods across multiple publishers. The volume of research robots being produced was found to steadily increase with time as the field has grown, this reflects on the necessity of pipe inspection as more networks become out-dated around the globe.

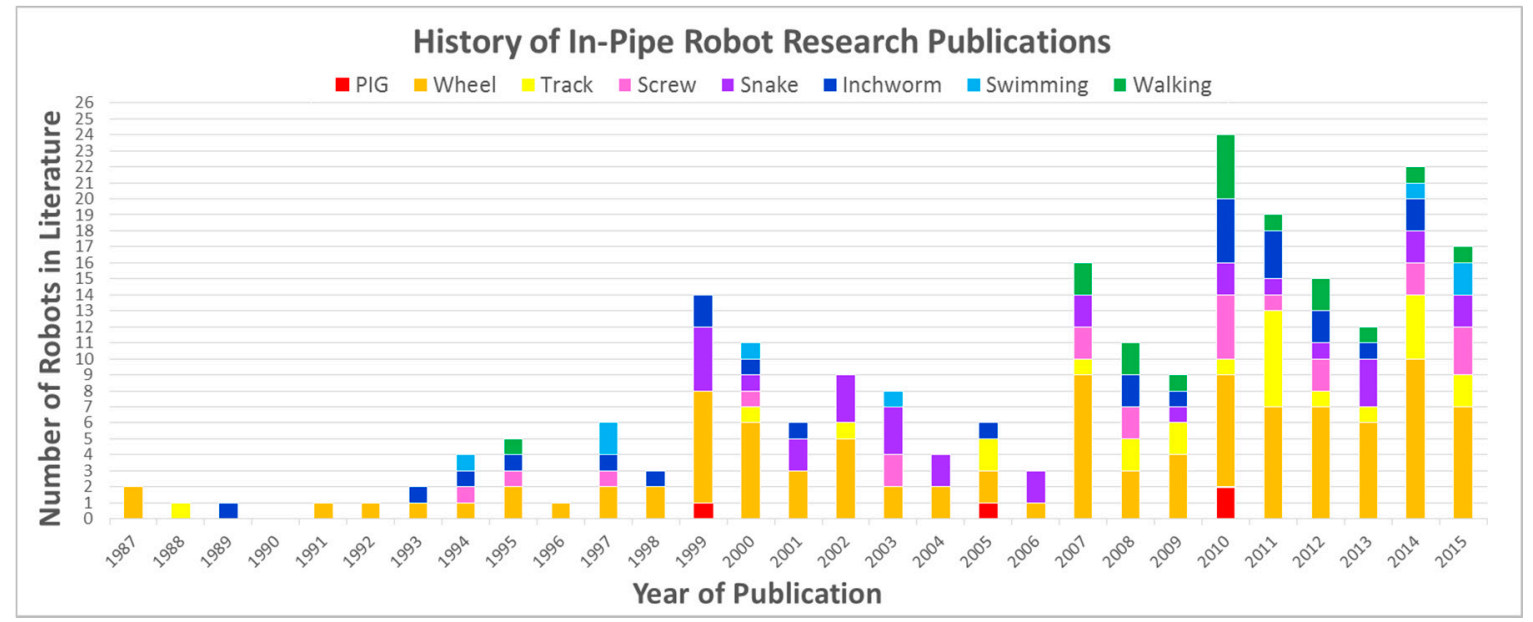

Figure 5. Locomotion types found in research based in-pipe robotic platforms.

Using the database of in-pipe research robots the key systems that have influenced the field have been selected and reviewed. These robotic platforms have been categorised in terms of their primary locomotion method, traction method, and their specified pipe diameter range. This section will look at robots from each of the eight in-pipe locomotion categories in detail. The strengths, weaknesses, and research direction of each type will be discussed later in Section 3.

\subsection{Pipeline Inspection Gauges}

PIGs may be simple devices but they are still the most efficient method of inspecting straight pipelines with a constant bore. New methods of pigging pipelines are still being developed, for example 
in 2005 the University of Durham developed a new form of "PIG" [6] which incorporates a propeller, driven by the fluid flowing in the pipe as a mobile power source. Brushes embedded in the chassis will only allow movement in a single direction depending on the direction the brushes are facing, this results in a steady motion either upstream or downstream.

\subsection{Wheeled Systems}

Classified by the use of wheels as a main locomotion method, these systems can be combined with any of the four traction methods. When combined with wall-press and magnetic traction methods simple wheel based systems become efficient in-pipe robots. Shows wheeled systems (orange) are the most prevalent method of in-pipe locomotion, being used in $43 \%$ of all systems. This is due to their adaptability and ease of combination with other locomotion types excluding tracks to create hybrid in-pipe systems. Wheeled systems are predominantly used with wall press traction methods, with $49 \%$ of all wall pressing robots using wheel locomotion.

MOGRER, an in-pipe robot developed by Niigata University circa 1987 was one of the first wall-pressing systems [7]. It was created for the purpose of industrial pipe inspection tasks and set out to solve the biggest problem at the time; climbing angled pipes against gravity. MOGRER was an improvement on an even earlier wall-press design FERRET-1 which introduced a three wheeled adaptable robot with passive configuration using a spring system [8]. MOGRER further improved the spring system forming a scissor structure similar to a pantograph mechanism, a popular choice for diametric change methods in the future. Advanced wall-pressing systems have been in development for over a decade, MRINSPECT is a wheeled in-pipe robot series designed at Sungkyunkwan University [9]. The robot is capable of performing all types of geometry manoeuvres besides valves and has a $50 \mathrm{~mm}$ adaptability range from 130 to $180 \mathrm{~mm}$. MRINSPECT uses a multi-axial differential gear system to control each of its four wheeled legs angles through active bevel drive connections.

Shenyang Institute of Automation have proposed a system which allows different robots to be created from the same platform. Although based around the same class size and pipe diameter of $200 \mathrm{~mm}$ three different systems were developed from one Multifunctional Mobile Unit, MMU [10]. These wheeled wall-pressing systems performed different functions; MMU1 could adapt to slight changes in pipe diameter, MMU2 was focused on detecting defects, MMU3 added a propeller function for fluid travel. The use of one common skeleton allowed these systems to be created from one design with relative ease compared a complete re-design. Advanced wheeled systems can adapt to almost all in-pipe obstacles, AQAM is a wheeled wall-pressing robot for 260-300 mm pipes, consisting of four arm mounted wheels in a single plane [11]. The robot has impressive manoeuvrability due to its four controllable arms and swivel mechanism to angle the wheels and hence rotate the robot in-pipe. This design allows the robot move through extremely complex in-pipe geometry such as T-Sections into a reduced vertical pipe section. AQAM shares its single plane design with Hanyang University's single-plane wheeled system [3]. These two systems have an advantage over multi-plane wall-pressing robots, by rotating in the pipe they can always maintains contact with the pipe walls in most complex obstacles. The problem faced with single plane contact is stability, any loss of wall contact in these designs will de-centralise the robot and make recovery extremely difficult. This is especially restrictive for Hanyangs system as the robot was capable of $80 \mathrm{~mm}-100 \mathrm{~mm}$ diameter pipes and hence only has a $20 \mathrm{~mm}$ adaptability range. Larger robots can also cover a wide range of diameters, Shanghai developed a large 3 planed wheeled system with a parallelogram mechanism that allowed adaption from $400 \mathrm{~mm}$ to $650 \mathrm{~mm}$ pipelines.

Wheeled in-pipe robots have capitalised on the ferrous properties of pipelines, magnetic robots allow the user to scale walls and maintain normal force without the need for wall-pressing functions. The first magnetic in-pipe robot was developed by the Osaka Gas Company in 1995, the system was a dual wheeled magnetic concept for the inspection 150-600 $\mathrm{mm}$ iron pipelines [12]. The concept was designed specifically to solve the T-section geometry problem without the need for a wall press robot, as these generally struggle with in-pipe valves and other sharp obstacle negotiation. MagneBike is 
an advanced example of a wheeled in-pipe robot using magnetic traction that can steer in a large range of in-pipe diameters, not only this but in-pipe obstacles such as T-Sections become trivial [13]. MagneBike could inspect a maximum pipeline diameter of $300 \mathrm{~mm}$ and was designed for industrial applications inspecting power plants. MagneBike's use of wall adhesion in-pipe reduces all complex in-pipe obstacles to either convex or concave corners which it must overcome. This simplified problem is outlined in other wheeled mobile magnetic robots, such as the hexagonal climbing robot from ALSTOM systems [14]. Other magnetic traction systems have developed locomotion methods that could be adapted in-pipe, Omni-Climber is a multi-directional magnetic wheeled robot with built in chassis compliance, allowing it to adapt to a wide range of pipe diameters [15]. This could be adapted to in-pipe scenarios allowing a small robot to explore pipes which are respectively large in diameter.

Wheeled systems are even used in conjunction with PIG-like locomotion; using the fluid force to accelerate. Kantaro a wheeled wall-pressing in-pipe robot for sewage pipe applications developed at Kyushu Institute of Technology, Japan takes a different approach to wall-pressing by combining it with a fluid driven locomotion [16]. Relying simply on pipe geometry to 'sit' in the pipe, whilst it also has adaptability built in to move from $200 \mathrm{~mm}$ to $300 \mathrm{~mm}$ diameters. Fully autonomous with passive damping springs and no tether, the system was ambitious, boasting the ability to manoeuvre through pipe bends without the need for a controller.

\subsection{Tracked Systems}

Track, or caterpillar based locomotion can be used in the place of wheels, they hold the advantage when generating friction. The large contact surface area makes these systems more stable but also generally larger than their wheeled counterparts. Caterpillar tracks have been used in $11 \%$ of in-pipe systems and are growing in popularity in research robotics, Figure 5 Tracked systems are also predominantly wall-pressing with $75 \%$ of all caterpillar robots using the traction method, the remaining $25 \%$ relying on gravity alone.

Famper was developed as a fully autonomous mobile pipeline exploration robot at Seoul National University [17], it was a track/caterpillar robot using wall-pressing traction with four-planes. Designed for 6-inch sewage pipes; Famper uses a passive method of wall-pressing to attain normal force against the pipe using compression springs and coupled with a slider linkage mechanism. The four separate caterpillar tracks can be actuated independently allowing the robot to differentially drive through elbows and other obstacles where varied speed is required. Famper performs well in 6-inch pipes and claims to be capable of T-Sections but has only been proven to complete vertical T-sections from a horizontal down, utilising gravity in its favour during the contact-loss phase of the manoeuvre. The full-bore design of Famper leaves little room for shape adaptability, with a full range of just $127-157 \mathrm{~mm}$, the purpose of which is mainly for obstacle negotiation. The lack of adaptability makes Famper unsuitable for any situation other than 6-Inch pipelines. This is a common occurrence with passive adaptability even in larger robots, systems such as AQAM which are exceptionally mobile and adaptable to changes in pipe geometry [11]. Caterpillar Wall-press systems are one of the most adaptive types of system in terms of shape adaptability. Tarbiat Modares University used active parallelogram adaption in a three-planed caterpillar wall-press system which could adapt from $250 \mathrm{~mm}$ to $350 \mathrm{~mm}$, however this could be increased by altering the length of the linkages [18]. The lead screw used to alter the height of the tracks keeps all three planes extended at the same rate and hence keeps the chassis central in the pipe. Nigata University's tracked robot uses an adapted scissor mechanism can passively adapt from $140 \mathrm{~mm}$ to $210 \mathrm{~mm}$ [19]. Hanyang University developed a two module caterpillar wall-pressing robot using differential steering and a passive adaptability module using a four bar mechanism to produce the required normal force on the tracks. This system could handle 80-100 mm pipes and can tackle many difficult in-pipe obstacles using the two-module design [20]. The separate tracked modules were connected via a tension spring which would drag the rear or front module through an obstacle, removing the need for both modules to steer. AGH University have prototyped a large in-pipe robot for the inspection of $200 \mathrm{~mm}$ pipelines that uses caterpillar tracks on a 
linkage which can alter their effective diameter [21]. This allows the robot to travel horizontally in a range of pipes from $201 \mathrm{~mm}$ to $235 \mathrm{~mm}$. Although vertical travel is possible, this is only the case when the tracks are fully extended essentially making vertical travel possible only in a $235 \mathrm{~mm}$ pipe. AGH's systems low adaptability range is in stark contrast with Ritsumeikan University's parallelogram crawler; a caterpillar wall-press system with an impressive adaptive diameter of $136 \mathrm{~mm}$ to $226 \mathrm{~mm}$ almost double its own length [22].

Paroys-II uses an actively controlled pantograph mechanism with a partially passive spring mechanism, this allows large changes to be controlled and small obstacles to be ignored [23]. Its use of a second set of articulated caterpillar tracks allows a huge adaptive range of 400-700 mm. Similarly Pukyong National University's tracked in-pipe robot uses modules with both passive and active adaptability mechanisms [24]. The shape adaptability is controlled using a driven threaded shaft lead screw and allows for transitions between $300 \mathrm{~mm}$ to $500 \mathrm{~mm}$ effective diameter by altering the position of a four bar linkage. The second module replaces the lead screw with a compression spring allowing for both passive and active diametric adaptions.

\subsection{Screw Systems}

Screw systems are defined by the method of locomotion in-pipe, using a rotary motion to drive themselves forward using a spiral track, to move through the pipe in a pitched circle. These systems are always wall-pressing as they rely on the inner walls in order to thread through the pipes, this allows them to climb vertical pipelines with ease. Screw locomotion robots are generally very difficult to back-drive due to their angled wheels or tracks, making them effective in high flow networks. Screw systems have stayed relatively steady in terms of growth in Figure 5 although the element is only used in $9 \%$ of research systems.

Heli-Pipe a screw wall-press system has a diametric adaptive ability of just $10 \mathrm{~mm}$, as a result four different prototypes were made, ranging from $170 \mathrm{~mm}$ to $40 \mathrm{~mm}$. The largest of the systems having a range of $10 \mathrm{~mm}$ and the smallest $5 \mathrm{~mm}$ [25]. Emerging methods of in-pipe adaptability show the possibility of continuously deforming systems with many degrees of freedom. SPRING is a screw type wall-press robot developed at Osaka University, although it relies on full wall traction it is unlike traditional full-bore wall-press systems which keep their chassis centralised in the pipe [26]. The design consists of many connected modules which form a continuous tight spiral some of which contain wheels allowing the robot to move in a spiral motion. When faced with a sharp change in diameter the robot can stretch this spiral increasing its pitch and therefore decreasing its diameter to less than half its optimal width of $150 \mathrm{~mm}$ pipelines. This function is entirely possible because of the lack of a centralised chassis, making the robot very adaptable when faced with obstacles such as valves. Current issues include the complexity of the design, optimal redesign of the robot is very difficult due to the amount of parameters involved and so a simulation tool had been created to aid in this. Shenyang University created a wall-press robot based on helix movement in-pipe, capable of $250 \mathrm{~mm}$ to $300 \mathrm{~mm}$ pipeline exploration using a passively adaptive four bar linkage [27]. The robot can complete complex manoeuvres such as T-Sections using its active drive module to steer the course.

\subsection{Snake Systems}

In-pipe snake robots are typically feature articulated joints in a modular design paired with wheels or tracks for locomotion. The articulation allows many degrees of freedom within a single system making them very versatile in their approach to obstacles. In the last 15 years, in-pipe snake robots have become the preferred method of commercial pipeline inspection and account for $13 \%$ of in-pipe robotic research.

The PipeTron series developed by HiBot, Tokyo is a multitude of robotic in-pipe exploration snake robots. Predominantly designed for tight bend systems such as refineries and chemical plants, the system is tethered for instant retrieval and consists of passively articulated wheels connected in a series [28]. The passive torsion springs in each module joint allow the robot to bend and alter shape 
depending on the problem encountered and the width of the pipe without requiring further actuators. Due to relatively low range of passive adaptability multiple platforms have been created for each commonly encountered pipe diameter: $75 \mathrm{~mm}, 100 \mathrm{~mm}$ and $150 \mathrm{~mm}$. The Explorer series is a prime example of the amount of effort going into translation of robots for different size pipelines, initially developed at Carnegie Mellon University [4]. This large snake robot is designed for the inspection of live gas networks under operating conditions, however it requires a full bore to operate with little adaptability ranging from just 150-200 $\mathrm{mm}$. Multiple systems have now been developed for operations in larger than specified networks.

Kanagawa University, presented an unusual and interesting method for coping with various pipe diameters. The robot is a hybrid of caterpillar and snake components, built from modular units each containing a driving caterpillar track [29]. Connecting three or more units allows the robot to drive in-pipe, should a larger diameter need to be traversed the number of driving units can be increased. Promising results were shown in experimental pipes of 100-300 mm, an exceptional range for a robot with modules of just $50 \mathrm{~mm}$. Czech Technical University, Prague have developed a snake robot with the purpose of identifying new methods of generating pipe contact without relying on traditional wall-press mechanisms such as pantographs and parallelogram linkages [30]. The modular snake robot consists of many segments each of which contain both rotary and translational actuators, this allows the snake to form structures much more complex than full bore robots. The shape adaptability of $100 \mathrm{~mm}$ is already greater than twice the length of the body segments which measure just $50 \mathrm{~mm}$, with greater numbers of segments this robot may even be able to handle a wider range of diameters. PIRATE was an in-pipe robot with the intended purpose of autonomous inspection of the gas distribution network in the Netherlands [31]. The robot is snake-like, and modular in nature featuring articulated clamping modules that can actively change the height of the robot to adapt to changes in pipe diameter. The design of the clamping modules was somewhat similar to the first proposal for a passively adaptive three wheeled inspection robot, [7]. However unlike passive systems the active articulated joints allow for an efficient change in pipe diameters, stretching to twice its original inspection diameter from $125 \mathrm{~mm}$ to $63 \mathrm{~mm}$.

\subsection{Walking Systems}

Walking type in-pipe robots use multi D.O.F. legs to move around the pipe, these are generally complex and quite large due to the number of actuators involved. Walking types which incorporate wall-pressing functions sacrifice mobility for increased stability, these are generally slower in-pipe. As a trade-off, the application of active wall pressing mechanisms give them a great amount of control over the applied normal force in pipe and they can deliver heavier payloads. Walking systems are somewhat uncommon with only $7 \%$ of research robots using the technique although they have been appearing more often in recent years as seen in Figure 5. MORITZ, a pipe inspection robot built at the Technical University of Munich was one of the first walking style robots to be developed [32]. Using 2 modules with a total of eight legs with 2 actuators each, a bending joint, and a rotation joint this complex system had a large number of degrees of freedom for an in-pipe robot. MORITZ was capable of travelling through $600 \mathrm{~mm}$ to700 $\mathrm{mm}$ diameter pipes thanks to the highly variable actuated legs, each with 2 degrees of freedom, extension of these legs allowed normal force to be controlled and frictional forces strong enough to hold up to $20 \mathrm{~kg}$ payloads. The high amounts of control and increased carrying capabilities compared to passive systems make robots such as MORITZ ideal for industrial inspection of power plants, where safety is a priority over speed of inspection and power requirements.

\subsection{Inchworm Systems}

In-pipe inchworms are currently only wall-pressing, they generate traction through large normal force applied at the front or back module whilst a central module contracts and extends in sequence. The high normal forces needed to support the robot during contraction makes these systems generally well suited for carrying high payloads. The use of point contact in inchworms and the removal of wheels or tracks makes them much more stable than other designs. Inchworms are also generally less 
prone to slip due to contaminants in-pipe as they can "cut" through the grease. Use of inchworms is quite widespread in the field, $11 \%$ of systems were found to use this technique in the study.

A highly mobile inchworm inspection robot based on a parallel manipulator, developed at YonSei University. This wall-press inspection device can handle 205-305 mm pipes the large inspection range coupled with its ability to traverse T-Sections makes up for the slow inspection speed [33]. These platforms have shown promise in industrial networks and have inspired the further exploration of large inchworm systems such as the 3SPR parallel manipulator under development at Yanshan University. This is a large two-module in-pipe inchworm robot, although currently in a theoretical state is intended to have large load carrying capacity for industrial applications [34]. Industrial pipeline networks often contain large amounts of oil and grease traces; it is these applications where wall-pressing systems such as these excel due to their low contact area end effectors. Case Western Reserve University have developed a new method of in-pipe robotic locomotion using continuous-wave peristalsis, the same mechanism employed by earthworms [35]. The robot, named CMMWorm, is composed of many interlinked joints forming a mesh like structure that can deform in the presence of an environmental change. The braided mesh can currently smoothly expand and contract from $220 \mathrm{~mm}$ to $180 \mathrm{~mm}$ and although currently unable to steer through in-pipe obstacles the mechanism shows great promise in shape adaptability research. These robots can potentially alter their effective diameters in-pipe in any section of their body.

\subsection{Propeller}

Propeller based locomotion is quite unusual in pipe inspection, used in combination with mainly fluid flow in water pipes this niche application featured in only $3 \%$ of reviewed systems. These water pipe inspection robots have the opportunity to utilise the fluid and develop swimming robots for use in leak detection. The main advantage of propeller systems is removing the need for wall contact altogether. A swimming in pipe robot was developed at the Massachusetts Institute of Technology as a solution to the challenges faced by robots relying on wall contact in water transport networks. The robot, MIT-MRL contains a chassis with housed electronics and two propellers for propulsion, the swimming nature of the robot allows exploration of pipes $100 \mathrm{~mm}$ and above autonomously [36]. The University of Sheffield took a different approach to swimming, developing a modular robot which can manipulate the fluid in order to generate propulsion in a certain direction by combining their chassis to form complex shapes. Each of the modules in this system form a section of a hydraulic system which when connected to another module forms a flowing network [37]. The Multifunctional Mobile Unit, MMU3 [6] also utilised a propeller in a version specified for fluid travel.

\subsection{Summary}

Table 1 provides a summary of robots reviewed in this section, where in-pipe geometry obstacles have been simplified to X (horizontal), Y (vertical), L (elbow), T (T-section), V (valves). The capability of the robot to traverse these obstacles is denoted by $(\boldsymbol{})$ when capable, $(X)$ when incapable, and (-) when a possibility in future versions of the system.

Table 1. Robot Type, Adaptability, and In-Pipe Capabilities.

\begin{tabular}{cccccccccccc}
\hline \multirow{2}{*}{ REF No. } & Information & \multicolumn{2}{c}{ Element } & \multicolumn{3}{c}{ Adaptability } & \multicolumn{4}{c}{ In-Pipe Geometry } \\
\cline { 2 - 12 } & Robot Name & Type 1 & Type 2 & Min & Max & Range & X & Y & L & T & V \\
\hline [13] & MagneBike & Magnetic & Wheel & 250 & $\infty$ & U & $\checkmark$ & $\checkmark$ & $\checkmark$ & $\checkmark$ & - \\
{$[37]$} & Fluid Modules & Modular & Swimming & 125 & $\infty$ & U & $\checkmark$ & $\checkmark$ & $\checkmark$ & $\checkmark$ & $\checkmark$ \\
{$[36]$} & MIT-MRL & Swimming & Swimming & 100 & $\infty$ & U & $\checkmark$ & $\checkmark$ & $\checkmark$ & $\checkmark$ & $\checkmark$ \\
{$[12]$} & Osaka Robot & Magnetic & Wheel & 150 & 600 & 450 & $\checkmark$ & $\checkmark$ & $\checkmark$ & $\checkmark$ & - \\
{$[23]$} & PAROYS-II & Caterpillar & Wall-Press & 400 & 700 & 300 & $\checkmark$ & $\checkmark$ & $\checkmark$ & $\checkmark$ & $\times$ \\
{$[7]$} & MOGRER & Wheel & Wall-Press & 520 & 800 & 280 & $\checkmark$ & $\checkmark$ & $\checkmark$ & $\times$ & $\times$ \\
{$[2]$} & Pukyong Robot & Wheel & Wall-Press & 300 & 500 & 200 & $\checkmark$ & $\checkmark$ & $\checkmark$ & - & $\times$ \\
\hline
\end{tabular}


Table 1. Cont.

\begin{tabular}{|c|c|c|c|c|c|c|c|c|c|c|c|}
\hline \multirow{2}{*}{ REF No. } & \multirow{2}{*}{$\begin{array}{l}\text { Information } \\
\text { Robot Name }\end{array}$} & \multicolumn{2}{|c|}{ Element } & \multicolumn{3}{|c|}{ Adaptability } & \multicolumn{5}{|c|}{ In-Pipe Geometry } \\
\hline & & Type 1 & Type 2 & Min & Max & Range & $X$ & $Y$ & L & $\mathrm{T}$ & $\mathbf{V}$ \\
\hline [29] & Kangawa Robot & Caterpillar & Snake & 100 & 300 & 200 & $\checkmark$ & $\checkmark$ & $\checkmark$ & $\checkmark$ & - \\
\hline [25] & HELI-PIPE Series & Screw & Wheel & 40 & 173 & 133 & $\checkmark$ & $\checkmark$ & $\checkmark$ & $x$ & $x$ \\
\hline [18] & Tarbiat Robot & Caterpillar & Wall-Press & 250 & 350 & 100 & $\checkmark$ & $\checkmark$ & $\checkmark$ & - & $x$ \\
\hline [34] & YonSei Robot & Inchworm & Inchworm & 205 & 305 & 100 & $\checkmark$ & $\checkmark$ & $\checkmark$ & - & $x$ \\
\hline [32] & MORITZ & Walking & Wall-Press & 600 & 700 & 100 & $\checkmark$ & $\checkmark$ & - & - & $x$ \\
\hline [16] & KANTARO & Wheel & Wall-Press & 200 & 300 & 100 & $\checkmark$ & $x$ & $\checkmark$ & $\checkmark$ & $x$ \\
\hline [31] & PIRATE & Wheel & Wall-Press & 41 & 125 & 84 & $\checkmark$ & $\checkmark$ & $\checkmark$ & - & $x$ \\
\hline [28] & PIPETRON I-VII & Snake & Wheel & 75 & 150 & 75 & $\checkmark$ & $\checkmark$ & $\checkmark$ & $\checkmark$ & $x$ \\
\hline [26] & SPRING & Screw & Wall-Press & 75 & 150 & 75 & $\checkmark$ & $\checkmark$ & $\checkmark$ & - & $x$ \\
\hline [4] & EXPLORER II & Snake & Wall-Press & 150 & 200 & 50 & $\checkmark$ & $\checkmark$ & $\checkmark$ & $\checkmark$ & $\checkmark$ \\
\hline [9] & MRINSPECT VI+ & Wheel & Wall-Press & 130 & 180 & 50 & $\checkmark$ & $\checkmark$ & $\checkmark$ & $\checkmark$ & $x$ \\
\hline [27] & Shenyang Robot & Wheel & Wall-Press & 250 & 300 & 50 & $\checkmark$ & $\checkmark$ & $\checkmark$ & - & $x$ \\
\hline [11] & AQAM & Wheel & Wall-Press & 259 & 305 & 46 & $\checkmark$ & $\checkmark$ & $\checkmark$ & $\checkmark$ & $x$ \\
\hline [35] & CMMWorm & Inchworm & Inchworm & 180 & 220 & 40 & $\checkmark$ & - & $\checkmark$ & - & $x$ \\
\hline [17] & FAMPER & Caterpillar & Wall-Press & 127 & 157 & 30 & $\checkmark$ & $\checkmark$ & $\checkmark$ & $\checkmark$ & $x$ \\
\hline [8] & FERRET-1 & Wheel & Wall-Press & 90 & 120 & 30 & $\checkmark$ & $\checkmark$ & $\checkmark$ & $x$ & $x$ \\
\hline [3] & Two-Plane Robot & Wheel & Wall-Press & 80 & 100 & 20 & $\checkmark$ & $x$ & $\checkmark$ & $\checkmark$ & $x$ \\
\hline [21] & AGH Robot & Caterpillar & Wall-Press & 210 & 210 & 0 & $\checkmark$ & $x$ & $\checkmark$ & $x$ & $x$ \\
\hline [30] & LOCOSNAKE & Snake & Snake & 120 & 120 & 0 & $\checkmark$ & $\checkmark$ & $\checkmark$ & $\checkmark$ & - \\
\hline
\end{tabular}

\section{Discussion}

Since the development of the first wall-pressing in-pipe robot in 1987 the technique has become the most popular method of generating traction in the field with over $23 \%$ of robots applying it in some way Figure 5. The popularity of wall-pressing systems stems from their ability to take advantage of the encompassing walls to generate traction. This feature is guaranteed in pipe networks and allows robots to easily move horizontally and even vertically thanks to the ability to generate friction in any orientation. Issues arise with wall-pressing robots when obstacles such as T-Sections present themselves and there is a possibility for contact loss. Systems were shown to have varying abilities depending on the number of points of contact with the wall varying between two, three or four planes of contact, examples of which are shown in Figure 4.

The field of in-pipe robotics research is heavily invested in the use of these classic wall-pressing systems, many of which have low diameter adaptability ranges. These systems are stable and efficient in their specified diameter range but none of the traditional systems reviewed showed the capability adapt to large changes. Systems such as AGH Universities $200 \mathrm{~mm}$ robot with only a $34 \mathrm{~mm}$ adaptability range would not be able to cope with even a $1.5 \times$ increase in pipe diameter and would lose the ability to climb angled pipe sections. In some cases, such as Hanyangs flat pipeline inspection robot a change over $20 \mathrm{~mm}$ would cause it to become completely unstable and unable to perform even basic locomotion tasks. Many of these robots would be deemed unsuitable for inspection of AGI networks. Some of these designs are simply too constrained by size due to their reliance on a chassis that is always concentric with the pipe. While the central mount provides a good base for adaptability mechanism control it does not benefit transformation range. This limitation means that pipe networks with large varying diameters cannot be inspected without an entire fleet of different class wall-press systems. Assuming a medium size wall-press robot with $100 \mathrm{~mm}$ adaptability range, a network with pipes ranging from $200 \mathrm{~mm}$ to $1000 \mathrm{~mm}$ diameters would require eight separate robots to inspect. Entering 8 different size systems into one network may require multiple access points on the pipe into the correct bore diameter and would need to be repeated across the AGI until the inspection is complete. 
The use of snake wall-pressing robots overcomes the limitations of traditional wall-pressing systems with multiple planar contact. Snake robots with wall-pressing capabilities can utilise articulated limbs to increase their effective diameter well over the range of parallelogram and pantograph mechanisms. For instance Kangawa University's snake system has an adaptability range of 100-300 mm [29]. Snake systems hold a great advantage over full-bore designs as they can be entered into smaller pipes as an access point into larger networks. This non full-bore design also allows them to pass through tricky obstacles such as valves which would stop most traditional wall-press systems in their tracks.

\section{Conclusions}

This review has presented a historical overview of the field as well as displaying the current in-pipe technology trends, with a specific emphasis on locomotion methods and their prevalence in the field. In-pipe robots are incredibly diverse and many different hybrid systems have been developed, some of which have fulfilled specific niches of pipe inspection. However, the inspection of highly varying diameter networks is an area that has not been fully explored, this task requires a method that is not constrained by pipe size. This is a complex issue and one that cannot be solved using full bore wall-pressing robots due to the limitations inherent in their adaptability.

We are coming close to $100 \%$ inspection of unpiggable pipelines the last $0.5 \%$ of network which is un-inspect-able using PIGs is difficult to deal with. In the coming years in-pipe robotic solutions will become available to inspect all networks of any diameter. Robotic technology is advancing and the field of in-pipe robotics is only growing with the increasing age of valuable pipelines around the globe. Ultimately small robotics systems that do not rely on wall-pressing for traction will be the solution as they allow access to both the smallest and largest diameter pipes in a network. Until then developments in the next 5 years may lead to vehicles with multiple traction methods e.g., a wall-press swimming hybrid such that it could negotiate both pipes containing fluid as well as non-running dry pipes. In the next 10 years the miniaturisation of robotic components and power sources may allow the design of micro-scale in-pipe systems. In the distant future mesoscale robots that flow within the pipes transport fluid may become the answer to the $100 \%$ inspection rate.

Acknowledgments: This work has been funded by National Grid Gas Transmission (NGGT) as part of Project GRAID: Gas Robotic Agile Inspection Device [2]. Gas Transmission and its project partners Synthotech [38], Premtech [39], and Pipeline Integrity Engineers [40] have been awarded £5.7 million in funding by the 2014 Network Innovation Competition to design and build an agile robotic platform to inspect buried pipework on high pressure gas sites. The robot will be designed to negotiate its own route around the sites with the ability to withstand pressures of up to 100 Barg.

Author Contributions: R. C. Richardson and A. E. Jackson have provided extensive supervision of the direction and scope of this work, and have contributed greatly to writing the paper.

Conflicts of Interest: The authors declare no conflict of interest.

\section{References}

1. Central Intelligence Agency. Pipelines. Available online: https://www.cia.gov/library/publications/theworld-factbook / fields/2117.html (accessed on 18 June 2017).

2. National Grid 2017. Project GRAID. Available online: http:/ / projectgraid.com/ (accessed on 14 June 2017).

3. Kwon, Y.-S.; Lee, B.; Whang, I.-C.; Kim, W.-K.; Yi, B.-J. A Flat Pipeline Inspection Robot with Two Wheel Chains. In Proceedings of the IEEE International Conference on Robotics and Automation (ICRA), Shanghai, China, 9-13 May 2011; pp. 5141-5146.

4. Schempf, H.; Mutschler, E.; Gavaert, A.; Skoptsov, G.; Crowley, W. Visual and Nondestructive Evaluation Inspection of Live Gas Mains Using the Explorer Family of Pipe Robots. J. Field Robot. 2010, 27, 217-249. [CrossRef]

5. Hertzberg, J.; Kirchner, F. Landmark-based autonomous navigation in sewerage pipes. In Proceedings of the First Euromicro Workshop on Advanced Mobile Robot, Kaiserslautern, Germany, 9-11 October 1996; pp. 68-73. 
6. Hu, Z.; Appleton, E. Dynamic Characterisitics of a Novel Self-Drive Pipeline PIG. Trans. Robot. 2005, 21, 781-789.

7. Okada, T.; Sanemori, T. MOGRER: A Vehicle Study and Realization for In-Pipe Inspection Tasks. J. Robot. Autom. 1987, 3, 573-582. [CrossRef]

8. Okada, T.; Kanade, T. A Three-Wheeled Self-Adjusting Vehicle in a Pipe, FERRET-1. Int. J. Robot. Res. 1987, 6, 60-75. [CrossRef]

9. Yang, S.U.; Kim, H.M.; Suh, J.S.; Choi, Y.S.; Mun, H.M.; Park, C.M.; Moon, H.; Choi, H.R. Novel Robot Mechanism Capable of 3D Differential Driving Inside Pipelines. In Proceedings of the IEEE International Conference on Intelligent Robots and Syst00ems, Chicago, IL, USA, 14-18 September 2014; pp. 1944-1949.

10. Li, P.; Ma, S.; Li, B.; Wang, Y. Multifunctional Mobile Units with a Same Platform for In-Pipe Inspection Robots. In Proceedings of the IEEE/RSJ International Conference on Intelligent Robots and Systems, Nice, France, 22-26 September 2008; pp. 2643-2648.

11. Lee, D.; Park, J.; Hyun, D.; Yook, G.; Yang, H.-S. Novel Mechanisms and Simple Locomotion Strategies for an In-Pipe Robot that can Inspect Various Pipe Types. Mech. Mach. Theory 2012, 56, 52-68. [CrossRef]

12. Kawaguchi, Y.; Yoshida, I.; Kikuta, T.; Yamada, Y. Internal Pipe Inspection Robto. In Proceedings of the IEEE International Conference on Robotics and Automation, Nagoya, Japan, 21-27 May 1995; pp. 857-862.

13. Tache, F.; Fischer, W.; Caprari, G.; Siegwart, R. MagneBike: A Magnetic Wheeled Robot with High Mobility for Inspecting Complex-Shaped Structures. J. Field Robot. 2009, 26, 453-476. [CrossRef]

14. Fischer, W.; Caprari, G.; Siegwart, R.; Moser, R. Very Compact Climbing Robot rolling on Magnetic Hexagonal Cam-Discs, with High Mobility on Obstacles but Minimal Mechanical Complexity. In Proceedings of the IEEE 6th German Conference on Robotics, Munich, Germany, 7-9 June 2010; pp. 1-7.

15. Tavakoli, M.; Viegas, C.; Marques, L.; Pires, J.N.; de Almeida, A.T. OmniClimbers: Omni-directional Magnetic Wheeled Climbing Robots for Inspection of Ferromagnetic Structures. Robot. Auton. Syst. 2013, 61, 997-1007. [CrossRef]

16. Nassiraei, A.A.F.; Kawamura, Y.; Ahrary, A.; Mikuriya, Y.; Ishii, K. Concept and Design of A Fully Autonomous Sewer Pipe Inspection Mobile Robot “KANTARO”. In Proceedings of the IEEE International Conference on Robotics and Automation, Roma, Italy, 10-14 April 2007; pp. 136-143.

17. Kim, J.-H.; Sharma, G.; Iyengar, S.S. FAMPER: A Fully Autonomous Mobile Robot for Pipeline Exploration. In Proceedings of the IEEE International Conference on Industrial Technology, Vina del Mar, Chile, 14-17 March 2010; pp. 517-523.

18. Moghaddam, M.M.; Arbabtafti, M.; Hadi, A. In-Pipe Inspection Crawler Adaptable to the Pipe Interior Diameter. Int. J. Robot. Autom. 2011, 26, 135-145. [CrossRef]

19. Oya, T.; Okada, T. Development of a steerable, wheel-type, in-pipe robot and its path planning. Adv. Robot. 2005, 19, 635-650. [CrossRef]

20. Kwon, Y.-S.; Yi, B.-J. Design and Motion Planning of a Two-Module Collaborative Indoor Pipeline Inspection Robot. Trans. Robot. 2012, 28, 681-696. [CrossRef]

21. Ciszewski, M.; Waclawski, M.; Buratowski, T.; Giergiel, M.; Kurc, K. Design, Modelling and Laboratory Testing of a Pipe Inspection Robot. Arch. Mech. Eng. 2015, 62, 395-408. [CrossRef]

22. Kakogawa, A.; Ma, S. Speed Analysis for three Modules of an In-Pipe Inspection Robots for Passing through Bent Pipes. In Proceedings of the IEEE International Conference on Robotics and Biometrics, Bali, Indonesia, 5-10 December 2014; pp. 1731-1736.

23. Park, J.; Hyun, D.; Cho, W.-H.; Kim, T.-H.; Yang, H.-S. Normal-Force Control for an In-Pipe Robot According to the Inclination of Pipelines. Trans. Ind. Electron. 2011, 58, 5304-5310. [CrossRef]

24. Min, J.; Setiawan, Y.D.; Pratama, P.S.; Kim, S.B.; Kim, H.K. Development and Controller Design of Wheeled-Type Pipe Inspection Robot. In Proceedings of the IEEE International Conference on Advances in Computing, Communications and Informatics, New Delhi, India, 24-27 September 2014; pp. 789-795.

25. Horodonica, M.; Preumont, A.; Burda, I.; Mignon, E. The Heli-Pipe Inspection Robots Architecture for Curved Pipes. In Proceedings of the IEEE International Conference of Manufacturing Systems, Crystal City Hyatt Regency Washington, DC, USA, 5-8 October 2003.

26. Nishihara, T.; Osuka, K.; Tamura, I. Development of a Simulation Model for Inner-Gas-Pipe Inspection Robot: SPRING. In Proceedings of the Society of Instrument and Control Engineers of Japan, Taipei, Taiwan, 18-21 August 2010. 
27. Ye, C.; Liu, L.; Xu, X.; Chen, J. Development of an In-Pipe Robot with Two Steerable Driving Wheels. In Proceedings of the IEEE International Conference on Mechatronics and Automation, Beijing, China, 2-5 August 2015.

28. Debenest, P.; Guarnieri, M.; Hirose, S. PipeTron Series-Robots for Pipe Inspection. In Proceedings of the IEEE 3rd International Conference on Applied Robotics for the Power Industry, Foz do Iguassu, Brazil, 14-16 October 2014; pp. 1-6.

29. Sato, K.; Ohki, T.; Lim, H.-O. Development of In-Pipe Robot Capable of Coping with Various Diameters. In Proceedings of the IEEE 11th International Conference on Control, Automation and Systems, Gyeonggi-do, Korea, 26-29 October 2011; pp. 1076-1081.

30. Prada, E.; Valasek, M.; Virgala, I.; Gmiterko, A.; Kelemen, M.; Hagara, M.; Liptak, T. New Approach of Fixation Possibilities Investigation for Snake Robot in the Pipe. In Proceedings of the IEEE International Conference on Mechanicals and Automation, Beijing, China, 2-5 August 2015.

31. Dertien, E.; Stramigioli, S.; Pulles, K. Development of an Inspection Robot for Small Diameter Gas Distribution Mains. In Proceedings of the IEEE International Conference on Robotics and Automation (ICRA), Shanghai, China, 9-13 May 2011; pp. 3447-3448.

32. Zagler, A.; Pfeiffer, F. "MORITZ" a Pipe Crawler for Tube Junctions. In Proceedings of the IEEE International Conference on Robotics and Automation, Taipei, Taiwan, 14-19 September 2003; pp. 2954-2959.

33. Jeon, W.; Park, J.; Kim, I.; Kang, Y.-K.; Yang, H. Development of High Mobility In-Pipe Inspection Robot. In Proceedings of the IEEE International Symposium on System Integration, Kyoto, Japan, 20-22 December 2011; pp. 479-484.

34. Lu, Y.; Yu, J.; Sui, C.; Han, J. Design of In-Pipe 3SPR/3RPS Parallel Manipulator and its Kinestatics Analysis. In Proceedings of the IEEE 5th Annual International Conference on Cyber Technology in Automation, Control and Intelligent Systems, Shenyang, China, 8-12 June 2015.

35. Boxerbaum, A.S.; Shaw, K.M.; Chiel, H.J.; Quinn, R.D. Continous Wave Peristaltic Motion in a Robot. Int. J. Robot. Res. 2012, 31, 302-318. [CrossRef]

36. Wu, Y.; Chatzigeorgiou, D.; Youcef-Toumi, K.; Zribi, M. Modeling and Parameter Estimation for In-pipe Swimming Robots. In Proceedings of the IEEE American Control Conference, Chicago, IL, USA, 1-3 July 2015; pp. 2007-2013.

37. Doyle, M.J.; Xu, X.; Gu, Y.; Perez-Diaz, F.; Parrott, C.; Grob, R. Modular Hydraulic Propulsion: A Robot that Moves by Routing Fluid Through Itself. In Proceedings of the IEEE International Conference on Robotics and Automation, Stockholm, Sweden, 16-21 May 2016; pp. 5189-5196.

38. Synthotech Limited. Available online: http://www.synthotech.com/ (accessed on 13 September 2017).

39. Premtech Limited. Available online: http:/ / www.premtechltd.com/ (accessed on 13 September 2017).

40. PIE-Pipeline Integrity Engineers. Available online: http:/ / www.pieuk.co.uk/ (accessed on 13 September 2017). 